\title{
Stability and Ensemble Inequivalence in a Globally Coupled System
}

\author{
M. Y. Choi ${ }^{1,2}$ and J. $\mathrm{Choi}^{3}$ \\ ${ }^{1}$ Department of Physics, Seoul National University, Seoul 151-747, Korea \\ ${ }^{2}$ Korea Institute for Advanced Study, Seoul 130-722, Korea \\ ${ }^{3}$ Department of Physics, Keimyung University, Taegu 704-701, Korea
}

\begin{abstract}
We consider a system of globally coupled rotors, described by a set of Langevin equations, and examine stability of the incoherent phase. The corresponding Fokker-Planck equation, providing a unified description of microcanonical and canonical ensembles, bears a few solutions, depending upon the ensemble. It is found that the stability of each solution varies differently with the temperature, revealing the inequivalence between the two ensembles. This also suggests a physical explanation of the quasi-stationarity observed in recent numerical results.
\end{abstract}

PACS numbers: 05.45.-a, 05.20.Gg, 05.40.-a, 64.60.Cn

A system of oscillators, coupled sinusoidally with each other, provides a prototype model for a variety of oscillatory phenomena in nature. With short-range coupling, i.e., nearest-neighbor interactions, such an oscillator system describes an array of Josephson junctions which has been a subject of extensive studies [1]. The opposite limit, corresponding to infinite-range interactions, leads to a system of globally coupled rotors, which simulates those systems having long-range forces in physics and biology. In spite of the mean-field character of such a system of globally coupled rotors, the system turned out to exhibit rich features in dynamical and statistical properties [2, 3, 4, 5, 6, 7]. Specifically, one can find an analytic solution in the canonical ensemble and the system has an equilibrium phase transition at a finite critical temperature in the ferromagnetic case. On the other hand, direct dynamical simulations reveal that it takes quite a long time for the system to reach the thermodynamic equilibrium [3, 6]. This anomalous diffusion phenomenon, dubbed quasi-stationarity, is observed only in microcanonical calculation, thus suggesting that there may exist inequivalence between microcanonical and canonical ensembles. Such a quasi-stationary state is believed to survive down well below the equilibrium critical temperature, attracting recently much attention together with controversy [ $\underline{5}, \mathbf{7}]$.

This work is to examine the stability of the incoherent (paramagnetic) phase in the system of globally coupled rotors and to provide an explanation as to the origin of the inequivalence between microcanonical and canonical ensembles. We consider the Fokker-Planck equation (FPE), which governs the time evolution of the system in both ensembles and thus provides a unified description, and obtain its stationary solutions, depending on the ensemble. The stability of each solution is probed and found to behave differently with the temperature. In particular, the microcanonical ensemble gives rise to additional incoherent solutions, which turn out to be neutrally stable even below the critical temperature. Such neutral stability may give rise to quasi-stationarity, thus suggesting a resolution of the controversy.
The dynamics of the coupled rotor system is governed by the set of equations of motion for the phase $\phi_{i}$ of the $i$ th rotor:

$$
M \ddot{\phi}_{i}+\sum_{j} J_{i j} \sin \left(\phi_{i}-\phi_{j}\right)=0
$$

where $M$ is the inertia of each rotor and $J_{i j}$ represents the coupling strength between rotors $i$ and $j$. With the introduction of the canonical momentum $p_{i}=M \dot{\phi}_{i}$, the above equations are transformed into a set of canonical equations with the Hamiltonian

$$
\mathcal{H}=\sum_{i} \frac{p_{i}^{2}}{2 M}-\sum_{i<j} J_{i j} \cos \left(\phi_{i}-\phi_{j}\right)
$$

on which the microcanonical description is based.

On the other hand, in the canonical description the system is in contact with a heat reservoir of temperature $T$ and described, in a most general way, by the set of Langevin equations:

$$
M \ddot{\phi}_{i}+\Gamma \dot{\phi}_{i}+\sum_{j} J_{i j} \sin \left(\phi_{i}-\phi_{j}\right)=\eta_{i},
$$

where $\Gamma$ is the damping coefficient and the Gaussian white noise $\eta_{i}(t)$ is characterized by the average $\left\langle\eta_{i}(t)\right\rangle=$ 0 and the correlation $\left\langle\eta_{i}(t) \eta_{j}\left(t^{\prime}\right)\right\rangle=2 \Gamma T \delta_{i j} \delta\left(t-t^{\prime}\right)$. It is straightforward from Eq. (3) to derive the FPE for the probability distribution $P\left(\phi_{i}, p_{i}, t\right)$ :

$$
\begin{aligned}
\frac{\partial P}{\partial t}=-\sum_{i} & \frac{p_{i}}{M} \frac{\partial P}{\partial \phi_{i}}+\sum_{i} \frac{\partial}{\partial p_{i}}\left[\frac{\Gamma}{M} p_{i}\right. \\
& \left.-\sum_{j} J_{i j} \sin \left(\phi_{i}-\phi_{j}\right)+\Gamma T \frac{\partial}{\partial p_{i}}\right] P .
\end{aligned}
$$

Note that Eq. (3) with $\Gamma$ set equal to zero reduces to Eq. (1), showing that Eq. (4) provides the starting point for both descriptions: the microcanonical one $(\Gamma=0)$ and the canonical one $(\Gamma \neq 0)$. In particular, the stationary solution of Eq. (4) is given by the canonical distribution 
$P^{(0)}\left(\phi_{i}, p_{i}\right) \propto e^{-\mathcal{H} / T}$ with the very Hamiltonian in Eq. (2) regardless of $\Gamma$ being zero or not.

In the case of global coupling $J_{i j}=J / N$, the set in Eq. (3) decouples into a single-particle equation

$$
M \ddot{\phi}+\Gamma \dot{\phi}+J \Delta \sin (\phi-\theta)=\eta,
$$

where the order parameter $\Delta e^{i \theta} \equiv N^{-1} \sum_{i}^{N} e^{i \phi_{i}}$ measures the emergence of coherence in the system and the rotor index $i$ has been suppressed. This in turn leads to the FPE for the single-rotor probability distribution $P(\phi, p, t)$ :

$\frac{\partial P}{\partial t}=-\frac{p}{M} \frac{\partial P}{\partial \phi}+\frac{\partial}{\partial p}\left[\frac{\Gamma}{M} p+J \Delta \sin (\phi-\theta)+\Gamma T \frac{\partial}{\partial p}\right] P$,

which reduces, in the absence of damping $(\Gamma=0)$, to the FPE for the microcanonical ensemble:

$$
\frac{\partial P}{\partial t}=-\frac{p}{M} \frac{\partial P}{\partial \phi}+J \Delta \sin (\phi-\theta) \frac{\partial P}{\partial p} .
$$

In terms of this probability distribution, the order parameter is simply given by $\Delta e^{i \theta}=\left\langle e^{i \phi}\right\rangle$, where $\langle\cdots\rangle$ denotes the average over the distribution $P(\phi, p, t)$. As pointed out for the general case, both Eqs. (6) and (7) support the same stationary solution $P^{(0)}(\phi, p)=\mathcal{Z}^{-1} e^{-\mathcal{H} / T}$ with the single-particle Hamiltonian

$$
\mathcal{H}=\frac{p^{2}}{2 M}-J \Delta \cos \phi,
$$

where the overall phase $\theta$ manifesting the $\mathrm{U}(1)$ symmetry has been absorbed into the definition of $\phi$. Note that $T$ is given in Eq. (6) but remains arbitrary for Eq. (7): In the microcanonical ensemble the temperature should be defined as a measure of the average kinetic energy according to $\left\langle p^{2}\right\rangle \equiv M T$. The partition function is determined by normalization: $\mathcal{Z}=(2 \pi)^{-1} \int d p \int d \phi e^{-\mathcal{H} / T}=$ $\sqrt{2 \pi M T} I_{0}(x)$, where $I_{0}(x)$ is the modified Bessel function of the zeroth order with $x \equiv J \Delta / T$.

This approach based on the FPE thus provides a unified description of microcanonical and canonical ensembles, and makes clear that both ensembles generate the same equilibrium behavior 2], described solely by the same stationary distribution $P^{(0)}(\phi, p)$. Namely, in both ensembles the equilibrium order parameter is given by

$$
\Delta=\int d p \int \frac{d \phi}{2 \pi} P^{(0)}(\phi, p) e^{i \phi}=\frac{1}{I_{0}(x)} \int \frac{d \phi}{2 \pi} e^{i \phi} e^{x \cos \phi},
$$

which, upon expanding $e^{x \cos \phi}$ in terms of the modified Bessel functions, yields

$$
\frac{T}{J} x=\frac{I_{1}(x)}{I_{0}(x)} .
$$

This determines whether the system exhibits coherence $(\Delta \neq 0)$ : The ordered phase emerges when $T / J$ is smaller than the slope of $I_{1}(x) / I_{0}(x)$ at $x=0$, and the ferromagnetic system $(J>0)$ undergoes a phase transition at the critical temperature $T_{c}=J / 2$.

We now turn our attention to the stability of the incoherent phase, which depends crucially on the ensemble employed, as shown below. For $\Delta=0$, the stationary solution $P^{(0)}(\phi, p)$ reduces to the Maxwell distribution, describing the incoherent phase. Unlike Eq. (6), however, Eq. (7), the FPE for the microcanonical ensemble, allows (incoherent) solutions of the general form: $P^{(0)}(\phi, p)=f_{0}(p)$, an arbitrary function of $p$, including the Maxwell distribution. Note that the uniformity in $\phi$ guarantees $\Delta=0$ since $\int d \phi e^{i \phi}=0$. To probe the stability of this stationary solution, we write $P(\phi, p, t)=$ $f_{0}(p)+f_{1}(\phi, p, t)$ and accordingly $\Delta(t)=\Delta_{0}+\Delta_{1}(t)$ [with $\Delta_{0}=0$ and $\left.\Delta_{1}(t)=\int d p \int \frac{d \phi}{2 \pi} f_{1}(\phi, p, t) e^{i \phi}\right]$ in Eq. (7), which leads to the stability equation

$$
\frac{\partial f_{1}}{\partial t}=-\frac{p}{M} \frac{\partial f_{1}}{\partial \phi}+J \Delta_{1} \sin \phi \frac{d f_{0}}{d p} .
$$

Since $f_{1}(\phi, p, t)$ and $\Delta_{1}(t)$ are periodic in $\phi$, one can Fourier decompose them into plane waves:

$$
\begin{aligned}
f_{1}(\phi, p, t) & =\sum_{k} \int d \omega e^{i(k \phi-\omega t)} \tilde{f}_{k}(p, \omega) \\
\Delta_{1}(t) & =\int d \omega e^{-i \omega t} \int d p \tilde{f}_{-1}(p, \omega) .
\end{aligned}
$$

Note here that the perturbed order parameter is proportional only to $\tilde{f}_{-1}(p, \omega)$. Substituting Eq. (11) into Eq. (10), one finds the following relations:

$$
\begin{aligned}
0= & \left(\omega-\frac{k p}{M}\right) \tilde{f}_{k}(p, \omega) \\
& -\frac{J}{2}\left[\int d p^{\prime} \tilde{f}_{-1}\left(p^{\prime}, \omega\right)\right]\left(\delta_{k, 1}-\delta_{k,-1}\right) \frac{d f_{0}}{d p},
\end{aligned}
$$

satisfied by the Fourier coefficients. Provided $\omega \neq k p / M$, it is obvious that all Fourier coefficients vanish except those corresponding to $k= \pm 1$. When $\omega=k p / M$, we have a continuous spectrum which correspond to a timedependent solution of the FPE with $\Delta=0$; this will be discussed later. For $k= \pm 1$, we divide Eq. (12) by $(\omega \mp p / M)$ and integrate over $p$, to obtain

$$
[1+\chi(\omega)] \int d p \tilde{f}_{-1}(p, \omega)=0,
$$

where the response function is given by

$$
\chi(\omega)=\frac{J M}{2} \int d p \frac{f_{0}^{\prime}(p)}{p+\tilde{\omega}}
$$

with $f_{0}^{\prime}(p) \equiv d f_{0} / d p$ and $\tilde{\omega} \equiv M \omega$. Accordingly, for a nontrivial solution, we must have $1+\chi(\omega)=0$. Since $\chi(\omega)$ in Eq. (14) has a simple pole at $p=-\tilde{\omega}$ on the complex $p$ plane on which the integration is performed, 
one needs to extend the frequency to complex values, i.e., $\tilde{\omega}=\tilde{\omega}_{r}+i \tilde{\omega}_{i}$. The appropriate analytic continuation then leads to the following relation, depending on the imaginary part of $\tilde{\omega}$ :

$$
\frac{2}{J M} \chi(\omega)= \begin{cases}\int_{-\infty}^{\infty} d p \frac{f_{0}^{\prime}(p)}{p+\tilde{\omega}} & \text { for } \omega_{i}>0 \\ \mathcal{P} \int_{-\infty}^{\infty} d p \frac{f_{0}^{\prime}(p)}{p+\tilde{\omega}}-i \pi f_{0}^{\prime}(-\tilde{\omega}) & \text { for } \omega_{i}=0 \\ \int_{-\infty}^{\infty} d p \frac{f_{0}^{\prime}(p)}{p+\tilde{\omega}}-2 i \pi f_{0}^{\prime}(-\tilde{\omega}) & \text { for } \omega_{i}<0\end{cases}
$$

where $\mathcal{P}$ stands for the principal part.

We thus have three different situations to determine the frequency: When $\omega_{i}>0$, the perturbation $\Delta_{1}(t)$ grows indefinitely to make the unperturbed solution $\Delta_{0}(=0)$ unstable. In this case the conditions for the real and the imaginary parts of the frequency to satisfy are

$$
\left\{\begin{array}{l}
\frac{2}{J M}+\int_{-\infty}^{\infty} d p \frac{\left(p+\tilde{\omega}_{r}\right) f_{0}^{\prime}(p)}{\left(p+\tilde{\omega}_{r}\right)^{2}+\tilde{\omega}_{i}^{2}}=0 \\
\int_{-\infty}^{\infty} d p \frac{f_{0}^{\prime}(p)}{\left(p+\tilde{\omega}_{r}\right)^{2}+\tilde{\omega}_{i}^{2}}=0
\end{array}\right.
$$

In the opposite case $\left(\omega_{i}<0\right)$, the perturbation dies out to make the system stable. The condition is then given by

$$
\left\{\begin{array}{l}
\frac{2}{J M}+\int_{-\infty}^{\infty} d p \frac{\left(p+\tilde{\omega}_{r}\right) f_{0}^{\prime}(p)}{\left(p+\tilde{\omega}_{r}\right)^{2}+\tilde{\omega}_{i}^{2}}+2 \pi \operatorname{Im} f_{0}^{\prime}(-\tilde{\omega})=0 \\
\tilde{\omega}_{i} \int_{-\infty}^{\infty} d p \frac{f_{0}^{\prime}(p)}{\left(p+\tilde{\omega}_{r}\right)^{2}+\tilde{\omega}_{i}^{2}}+2 \pi \operatorname{Re} f_{0}^{\prime}(-\tilde{\omega})=0 .
\end{array}\right.
$$

Finally, when $\omega_{i}=0$, the condition simply reads

$$
\left\{\begin{array}{l}
\frac{2}{J M}+\mathcal{P} \int_{-\infty}^{\infty} d p \frac{f_{0}^{\prime}(p)}{p+\tilde{\omega}_{r}}=0 \\
f_{0}^{\prime}\left(-\tilde{\omega}_{r}\right)=0
\end{array}\right.
$$

and the system is characterized by the neutral stability, oscillating with frequency $\omega_{r}$. When this condition is met, the system may stay in this state rather a long time presumably until higher-order nonlinear terms come into play and break neutrality.

We are now ready to discuss the stability of the incoherent phase associated with various solutions of the FPE: the uniform distribution and the rotating distribution as well as the Maxwell distribution; the first two exist only for the microcanonical ensemble while the third for both ensembles.

Uniform distribution. In this simplest case, momenta are distributed uniformly in the range $[-\alpha, \alpha]$, i.e., $f_{0}(p)=1 / 2 \alpha$ for $-\alpha<p<\alpha$. Known as a waterbag distribution, this has been adopted in most of dynamical calculations performed so far. Substitution of $f_{0}^{\prime}(p)=(2 \alpha)^{-1}[\delta(p+\alpha)-\delta(p-\alpha)]$ into Eqs. (15) - (17), depending on the sign of $\omega_{i}$, leads to

$$
\begin{aligned}
& \omega_{i}= \pm \sqrt{\frac{J}{2 M}-\left(\frac{\alpha}{M}\right)^{2}}, \quad \omega_{r}=0 \text { for } \alpha<\alpha_{1} \\
& \omega_{r}= \pm \sqrt{\left(\frac{\alpha}{M}\right)^{2}-\frac{J}{2 M}}, \quad \omega_{i}=0 \text { for } \alpha>\alpha_{1},
\end{aligned}
$$

which indicates that the frequency of $\Delta_{1}(t)$ and $f_{1}(t)$ has only the imaginary/real part for $\alpha$ smaller/larger than $\alpha_{1} \equiv \sqrt{J M / 2}$. Recalling the definition of the temperature in the microcanonical description, we have $T=\left\langle p^{2}\right\rangle / M=\alpha^{2} / 3 M$, from which we find $T_{1}=J / 6$. At low temperatures $\left(T<T_{1}\right)$ the frequency of the perturbation has the imaginary part, giving rise to the growth (as well as decay) of the perturbation. Accordingly the solution is unstable. However, at temperatures $T>T_{1}$, the system oscillates with the (real) frequency, and neither dissipation nor growth can be observed. We point out here that $T_{1}$ above which the neutral stability emerges is well below the mean field critical temperature $T_{c}=J / 2$. This is precisely what has been observed in various numerical simulations based on the microcanonical ensemble: Starting from the incoherent initial configuration $\left(\Delta_{0}=0\right)$ with the uniform distribution, many authors reported that the system remains in the incoherent state for a long time, before it eventually falls into the coherent state $\left(\Delta_{0} \neq 0\right)$. Such quasi-stationarity persists down well below $T_{c}[\underline{8}]$, but the present analysis shows that it ceases to exist below $T_{1}$.

Maxwell distribution. We next consider the Maxwell distribution, $f_{0}(p)=(2 \pi M T)^{-1 / 2} e^{-p^{2} / 2 M T}$. For $\omega_{i}>0$, substituting $f_{0}^{\prime}(p)=-(p / M T) f_{0}(p)$ into Eq. (15) and noting $f_{0}^{\prime}(-p)=-f_{0}^{\prime}(p)$, we find that $\omega_{r}=0$ is clearly a solution of the second one in Eq. (15). From the first of Eq. (15), we obtain

$$
1-\frac{2 T}{J}=\sqrt{\pi} y e^{y^{2}} \operatorname{erfc}(y),
$$

where $\operatorname{erfc}(y)$ is the complementary error function with $y \equiv \sqrt{M / 2 T} \omega_{i}$. Since the right-hand side is monotonically increasing from zero to unity as $y$ grows from zero, there is one real solution present for $T<J / 2$ and no solution for $T>J / 2$. This implies that the system is unstable for $T<J / 2$ due to the presence of the solution $\omega_{i}>0$. For $\omega_{i}<0$, we first note

$$
f_{0}^{\prime}\left(\tilde{\omega}_{r}+i \tilde{\omega}_{i}\right)=-\frac{\tilde{\omega}_{r}+i \tilde{\omega}_{i}}{\sqrt{2 \pi M^{3} T^{3}}} e^{-\left(\tilde{\omega}_{r}^{2}-\tilde{\omega}_{i}^{2}\right) / 2 M T} e^{-i \tilde{\omega}_{r} \tilde{\omega}_{i} / M T},
$$

from which we see $\operatorname{Re} f_{0}^{\prime}\left(\tilde{\omega}_{r}+i \tilde{\omega}_{i}\right)=0$ for $\tilde{\omega}_{r}=0$ while $\operatorname{Im} f_{0}^{\prime}\left(i \tilde{\omega}_{i}\right)=-\left(2 \pi M^{3} T^{3}\right)^{-1 / 2} \omega_{i} e^{\tilde{\omega}_{i}^{2} / 2 M T}$. This suggests that $\tilde{\omega}_{r}=0$ is again a solution of the second equation in Eq. (16) whereas the first one reduces to

$$
\frac{2 T}{J}-1=\sqrt{\pi}|y| e^{y^{2}}[2-\operatorname{erfc}(|y|)] .
$$


Similarly to the $\omega_{i}>0$ case, the right-hand side of the above equation is monotonically increasing with $|y|$, rendering a solution $\omega_{i}<0$ only for $T>J / 2$. Finally, when $\omega_{i}=0$, we have also $\omega_{r}=0$ from $f_{0}^{\prime}\left(-\tilde{\omega}_{r}\right)=0$; this trivial solution appears at $T=J / 2$, seen from the first one of Eq. (17). We thus conclude that the Maxwell distribution, leading to the incoherent phase at $T>T_{c}=J / 2$, becomes unstable below $T_{c}$, where coherence develops.

This conclusion holds also for the canonical ensemble, where the Maxwell distribution is the only stationary distribution: Equation (6) leads to the stability equation in the form of Eq. (10) with the additional term depending on $\Gamma$; this, however, has no effects on the perturbation in $\phi$, giving rise to the same instability below $T_{c}$.

Rotating distribution. In addition to the timeindependent solutions discussed so far, Eq. (7) also possesses a time-dependent solution of the general form $P^{(0)}(\phi, p, t)=u(\phi-p t / M)$ for $\Delta=0$. In this rotating solution the phase grows continuously with a continuous spectrum as mentioned previously. Requiring periodicity in $\phi$, we write

$$
P^{(0)}(\phi, p, t)=\sum_{k} e^{i k\left(\phi-\frac{p}{M}\right) t} F_{k}(p),
$$

where $F_{k}(p)$ is an arbitrary function except for $F_{ \pm 1}(p)=$ 0 due to $\Delta=0$. The stability analysis for this solution is entirely similar to that of stationary solutions and will not be repeated here. The response function is given by

$$
\chi(\omega)=\frac{J M}{2} \int d p\left[\frac{F_{0}^{\prime}(p)}{p+\tilde{\omega}}-\frac{F_{-2}^{\prime}(p)}{p-\tilde{\omega}}\right]
$$

in place of Eq. (14), and appropriate changes should be made in the subsequent equations through (17). Although one can consider various distributions for $F_{k}$ [9], we only quote the results from uniform distributions for $F_{0}(p)$ and $F_{-2}(p)$, with the distribution width $2 \alpha$ as before:

$$
\begin{aligned}
& \omega_{i}= \pm \sqrt{\frac{J}{M}-\left(\frac{\alpha}{M}\right)^{2}}, \quad \omega_{r}=0 \text { for } \alpha<\alpha_{2} \\
& \omega_{r}= \pm \sqrt{\left(\frac{\alpha}{M}\right)^{2}-\frac{J}{M}}, \quad \omega_{i}=0 \text { for } \alpha>\alpha_{2}
\end{aligned}
$$

with $\alpha_{2} \equiv J / M$. Again, one can relate the average kinetic energy with the temperature: $T=\left\langle p^{2}\right\rangle / M=$ $\alpha^{2} / 3 M$, from which one has $T_{2}=\alpha_{2}^{2} / 3 M=J / 3$. Thus the rotating solution has neutral stability for $T>T_{2}$ and becomes unstable below $T_{2}$. Note once again that $T_{2}$ is lower than the equilibrium critical temperature $T_{c}$.

Table I summarizes our results of the stability analysis for various solutions, depending on the temperature. While coherence develops in equilibrium below the transition temperature $T_{c}=J / 2$, the dynamic behavior of the incoherent phase depends on the ensemble: In the canonical ensemble the Maxwell distribution is the only

\begin{tabular}{|cccc|}
\hline & Maxwell & uniform & rotating \\
\hline$J / 2<T$ & $\mathrm{~s}$ & $\mathrm{n}$ & $\mathrm{n}$ \\
$J / 3<T<J / 2$ & $\mathrm{u}$ & $\mathrm{n}$ & $\mathrm{n}$ \\
$J / 6<T<J / 3$ & $\mathrm{u}$ & $\mathrm{n}$ & $\mathrm{u}$ \\
$T<J / 6$ & $\mathrm{u}$ & $\mathrm{u}$ & $\mathrm{u}$ \\
\hline
\end{tabular}

TABLE I: Stability of the incoherent phase for various distributions. Whereas the Maxwell distribution corresponds to both ensembles, the other two correspond only to the microcanonical ensemble. The letters $\mathrm{s}, \mathrm{n}$, and $\mathrm{u}$ stand for stable, neutrally stable, and unstable, respectively.

stationary distribution corresponding to the incoherent phase and becomes unstable below $T_{c}$. On the other hand, the microcanonical ensemble gives rise to additional distributions such as the uniform and the rotating one, which remain neutrally stable even below $T_{c}$. Putting these together, we are led to the plausible conclusion that the quasi-stationarity found in numerical simulations below $T_{c}$ has its origin in the neutrally stable solutions in the microcanonical ensemble and will not appear in the canonical ensemble.

Similar calculations for the antiferromagnetic system (with $J<0$ ), where coherence does not emerge [see Eq. (9)], lead to that all three types of solutions are always neutrally stable [9]. This may give a clue to nature of the non-stationary behavior observed recently [10].

M.Y.C. thanks the CNRS at Université Louis Pasteur for hospitality, where part of this work was accomplished. This work was supported in part by the Overhead Research Fund of SNU and by the Basic Research Program (Grant No. R01-2002-000-00285-0) of KOSEF.

[1] See, e.g., M.Y. Choi and D.J. Thouless, Phys. Rev. B 64, 014305 (2001) and references therein.

[2] M. Antoni and S. Ruffo, Phys. Rev. E 52, 2361 (1995); T. Dauxois, V. Latora, A. Rapisarda, S. Ruffo, and A. Torcini, e-print cond-mat/0208456

[3] V. Latora, A. Rapisarda, and S. Ruffo, Phys. Rev. Lett. 83, 2104 (1999).

[4] H. Hasegawa, e-print cond-mat/0210473

[5] M. A. Montemurro, F. A. Tamarit, and C. Anteneodo, e-print cond-mat/0205355

[6] Y. Yoshiyuki, e-print nlin CD/0209031.

[7] D. Zanette and M. A. Montemurro, e-print cond-mat/0209640

[8] Numerical experiments usually use the internal energy $U$ as the control parameter, which is related with the temperature $T$ via $U=T / 2+\left(1-\Delta^{2}\right) / 2$ for $J=1$. We here use a slightly different definition of the potential energy.

[9] M. Y. Choi and J. Choi, unpublished.

[10] J. Barré, F. Bouchet, T. Dauxois, S. Ruffo, Phys. Rev. Lett. 89, 110601 (2002). 